\title{
Study of relativistic bound states in a scalar model using diagonalization/Monte Carlo methods
}

\author{
Buḡra Borasoy ${ }^{a *}$ and Dean Lee ${ }^{b \dagger}$ \\ ${ }^{a}$ Physik Department, Technische Universität München, D-85747 Garching, Germany \\ ${ }^{b}$ Dept. of Physics, Univ. of Massachusetts, Amherst, MA 01003, U.S.A.
}

November 19, 2018

\begin{abstract}
We use a recently proposed diagonalization/Monte Carlo computational scheme to study relativistic two-body and three-body bound states in $\left(\phi^{6}-\phi^{4}\right)_{1+1}$ theory. We find that the approach is well-suited for calculating bound state energies and wavefunctions.
\end{abstract}

\section{Introduction}

The structure and phenomenology of relativistic bound states are of primary interest to particle and nuclear physics. Unfortunately even in the simplest field theory models our understanding of relativistic bound states is limited by a number of complications. Monte Carlo simulation on the lattice is the standard tool for determining particle masses. One can find the low energy spectrum from the decay constants of Euclidean correlation functions. Though the procedure is generally successful it becomes more difficult and less reliable if the state of interest has the same quantum numbers as another lower energy state. Complications also arise for non-confining interactions, where states just above the continuum threshold can obscure the signal of the bound state. Both of these problems arise even in seemingly simple systems such as we consider here, namely, two-body and three-body bound states in $\left(\phi^{6}-\phi^{4}\right)_{1+1}$.

One remaining complication is of a more operational nature, and it concerns the interface between computational methods and phenomenology. Much of the interesting and important physics associated with bound states is contained in subtle details. A measurement of gross properties such as the binding energy

\footnotetext{
*borasoy@physik.tu-muenchen.de

†dlee@physics.umass.edu
} 
is a necessary first step, but one also hopes to gain more descriptive information like the structure of the wavefunction, its response to various interactions, or point-by-point comparisons with intuitive non-relativistic models.

These issues were part of the motivation for a recently proposed approach to computational quantum problems which combines diagonalization and Monte Carlo techniques [1, 2]. Diagonalization makes it possible to consider systems with sign oscillations and extract detailed information about wavefunctions and excited states, while Monte Carlo allows one to handle the exponential increase in the number of basis states for large volume systems. The first half of the method involves finding and diagonalizing the Hamiltonian restricted to an optimal subspace. This subspace is designed to include the most important basis vectors of the lowest energy eigenstates. Once the most important basis vectors are found and their interactions treated exactly, Monte Carlo is used to sample the contribution of the remaining basis vectors. In this paper we demonstrate how the new diagonalization/Monte Carlo scheme is applied to the study of relativistic bound states.

The essential problem of the lattice Monte Carlo approach to bound states near the continuum threshold involves finding the inverse Laplace transform of two-point correlation functions at energies close to thresholds where the spectral density is large. This is usually done by a non-linear multi-parameter fit of twopoint correlators for several different operators. While in principle one might reach arbitrary accuracy by adding more parameters to the fit, in practice the presence of even very small stochastic error will destabilize the fit and imposes an upper limit on the accuracy. The main advantage of our method, therefore, is not in computing speed but in numerical stability. Our method also provides additional information about the wavefunction of the bound state. These two statements are not unrelated. Our method has greater numerical stability because we are implicitly using the information contained in the wavefunctions to separate states. It is also worthwhile noting that in two dimensions the diagonalization/Monte Carlo approach can be applied within the framework of discretized light cone and might prove useful since, aside from zero-mode problems, the exponential growth of the number of basis states for large volume systems has so far provided a limit to discretized light cone.

The organization of the paper is as follows. We begin with a discussion of the $\phi^{6}-\phi^{4}$ Hamiltonian in $1+1$ dimensions and its decomposition into momentum modes. We then review the basic features of quasi-sparse eigenvector diagonalization and stochastic error correction. In the following two sections we briefly set aside the numerical discussion and derive solutions to the nonrelativistic versions of the two-body and three-body bound state problems. We then compare our numerical results for the relativistic bound states energies and wavefunctions with an assortment of different approximations and results from the literature. 


\section{Fock states and the Hamiltonian}

Our starting point is the Hamiltonian density for $\phi^{6}-\phi^{4}$ theory in $1+1$ dimensions,

$$
\mathcal{H}=: \frac{1}{2}\left(\frac{\partial \phi}{\partial t}\right)^{2}+\frac{1}{2}\left(\frac{\partial \phi}{\partial x}\right)^{2}+\frac{\mu^{2}}{2} \phi^{2}+\lambda\left(\phi^{6}-\phi^{4}\right):,
$$

where normal ordering is with respect to the mass $\mu$. This theory was introduced in [3] as a simple model with a stable bound state. In $1+1$ dimensions, normal ordering is sufficient to renormalize the theory.

We will consider the theory in a periodic box of length $2 L$. This allows us to expand in free particle momentum modes and rewrite the Hamiltonian in terms of mode creation and annihilation operators [1], 5],

$$
\begin{aligned}
H & =\sum_{n} \omega_{n}(\mu) a_{n}^{\dagger} a_{n}-\frac{\lambda}{8 L} \sum_{n_{1}+\cdots+n_{4}=0}: \frac{\left(a_{n_{1}}+a_{-n_{1}}^{\dagger}\right)}{\sqrt{\omega_{n_{1}}(\mu)}} \cdots \frac{\left(a_{n_{4}}+a_{-n_{4}}^{\dagger}\right)}{\sqrt{\omega_{n_{4}}(\mu)}}: \\
& +\frac{\lambda}{32 L^{2}} \sum_{n_{1}+\cdots+n_{6}=0}: \frac{\left(a_{n_{1}}+a_{-n_{1}}^{\dagger}\right)}{\sqrt{\omega_{n_{1}}(\mu)}} \cdots \frac{\left(a_{n_{6}}+a_{-n_{6}}^{\dagger}\right)}{\sqrt{\omega_{n_{6}}(\mu)}}: .
\end{aligned}
$$

In (2) we have used

$$
\omega_{n}(\mu)=\sqrt{\frac{n^{2} \pi^{2}}{L^{2}}+\mu^{2}} .
$$

We can represent any momentum-space Fock state as a string of occupation numbers, $\left|o_{-} N_{\max }, \cdots, o_{N_{\max }}\right\rangle$, where

$$
a_{n}^{\dagger} a_{n}\left|o_{-N_{\max }}, \cdots, o_{N_{\max }}\right\rangle=o_{n}\left|o_{-N_{\max }}, \cdots, o_{N_{\max }}\right\rangle .
$$

For the calculations presented here, we set the length of the box to size $L=$ $2.5 \pi \mu^{-1}$ and restrict our attention to momentum modes such that $|n| \leq N_{\max }$, where $N_{\max }=10$. This corresponds with a momentum cutoff scale of $\Lambda=4 \mu$. A change in the cutoff $N_{\max }$ will lead to adjustments in $\mu$ and $\lambda$ in order to keep the physics independent of $N_{\max }$. However, these corrections begin at $\mathcal{O}\left(1 / N_{\max }^{2}\right)$ as can be checked by power counting. The dependence on $N_{\max }$ has been measured explicitly for the bound state data presented in this paper. It is considerably smaller than the dominant error, which is due to higher order corrections in the series stochastic error method, see below.

\section{QSE diagonalization}

In order to find approximate eigenvalues and eigenvectors of the Hamiltonian in (2), we use a method called quasi-sparse eigenvector (QSE) diagonalization. QSE diagonalization is an iterative algorithm that finds the most important basis vectors of the low energy eigenstates for a quantum Hamiltonian. We follow closely the discussion presented in 11]. We start with a complete basis for which the Hamiltonian matrix $H_{i j}$ is sparse. In our case we use the momentumspace Fock basis. The QSE method involves the following iterated steps: 
1. Select a subset of basis vectors $\left\{e_{i_{1}}, \cdots, e_{i_{n}}\right\}$ and call the corresponding subspace $S$.

2. Diagonalize $H$ restricted to $S$ and find one eigenvector $v$.

3. Sort the basis components of $v$ according to their magnitude and remove the least important basis vectors.

4. Replace the discarded basis vectors by new basis vectors. These are selected at random from a pool of candidate basis vectors which are connected to the old basis vectors through non-vanishing matrix elements of $H$.

5. Redefine $S$ as the subspace spanned by the updated set of basis vectors and repeat steps 2 through 5 .

If the subset of basis vectors is sufficiently large, the exact low energy eigenvectors will be stable fixed points of the update process. This is the single eigenvector version of the algorithm. The multiple eigenvector version simply involves selecting more than one eigenvector each iteration and retaining the most important basis vectors for each of the eigenvectors. One of the most attractive features of our method is that we are working directly with the infinite dimensional Hilbert space. There is no restriction on the maximum occupation for any mode. This is a clear advantage over standard diagonalization methods such as Lanczos.

In this analysis we work in the rest frame of the bound state and consider Fock states with total momentum zero. Due to $\phi \rightarrow-\phi$ reflection symmetry we can diagonalize the even and odd $\phi$ sectors separately. We use the multiple eigenvector algorithm to obtain the four lowest eigenstates in the even sector and in the odd sector For each QSE iteration, we select 50 new basis vectors and keep 400 basis vectors. The final QSE results are used as the starting point for the stochastic error correction method.

\section{Stochastic error correction}

The QSE method approximates the low energy eigenvalues and eigenvectors of the Hamiltonian by finding and diagonalizing optimized subspaces $S$. This process however has an intrinsic error due to the omission of basis states not in $S$. The goal of stochastic error correction is to sample the contribution of these remaining basis vectors. In the present investigation we use the so-called series expansion method up to first order. This has been explained in detail in [2, 1] and therefore we restrict ourselves to the review of basic formulae.

Let $\lambda_{i}$ be an eigenvalue and $\left|v_{i}\right\rangle$ be the corresponding eigenvector of $H$ restricted to the subspace $S$ (the span of the subset of basis vectors after step

\footnotetext{
${ }^{1}$ We have found only one two-body bound state and one three-body bound state. Thus in a follow-up study one could suffice with fewer states in each sector.
} 
3 of the QSE algorithm). The remaining basis vectors in the full space not contained in $S$ will be denoted by $\left|A_{i}\right\rangle$, and we are interested in sampling the contribution of these vectors. We assume that $\lambda_{1}$ and $\left|v_{1}\right\rangle$ are close to the corresponding quantities for the full Hamiltonian $H$. If we now expand in powers of

$$
\left(\left\langle A_{i}|H| A_{i}\right\rangle-\lambda_{1}\right)^{-1}
$$

we find that the first order correction to $\lambda_{1}$ is

$$
\delta \lambda_{1}=-\sum_{i} \frac{\left\langle v_{1}|H| A_{i}\right\rangle\left\langle A_{i}|H| v_{1}\right\rangle}{\left\langle A_{i}|H| A_{i}\right\rangle-\lambda_{1}}
$$

and the first order correction to $\left|v_{1}\right\rangle$ is

$$
\left|\delta v_{1}\right\rangle=-\sum_{i} \frac{\left|A_{i}\right\rangle\left\langle A_{i}|H| v_{1}\right\rangle}{\left\langle A_{i}|H| A_{i}\right\rangle-\lambda_{1}}+\sum_{j \neq 1} \sum_{i} \frac{\left|v_{j}\right\rangle\left\langle v_{j}|H| A_{i}\right\rangle\left\langle A_{i}|H| v_{1}\right\rangle}{\left(\lambda_{j}-\lambda_{1}\right)\left(\left\langle A_{i}|H| A_{i}\right\rangle-\lambda_{1}\right)} .
$$

The sum over all vectors $\left|A_{i}\right\rangle$ is too unwieldy to perform exactly. Instead we sample the sum stochastically in the following manner. Let $P\left(A_{\operatorname{trial}(i)}\right)$ be the probability of selecting $\left|A_{\operatorname{trial}(i)}\right\rangle$ on the $i^{\text {th }}$ trial. Then

$$
\delta \lambda_{1}=-\lim _{N \rightarrow \infty} \frac{1}{N} \sum_{i=1, \ldots, N} \frac{\left\langle v_{1}|H| A_{\text {trial }(i)}\right\rangle\left\langle A_{\text {trial }(i)}|H| v_{1}\right\rangle}{\left(\left\langle A_{\text {trial }(i)}|H| A_{\text {trial }(i)}\right\rangle-\lambda_{1}\right) P\left(A_{\text {trial }(i)}\right)}
$$

and

$$
\begin{aligned}
\left\langle w \mid \delta v_{1}\right\rangle & =-\lim _{N \rightarrow \infty} \frac{1}{N} \sum_{i} \frac{\left\langle w \mid A_{\text {trial }(i)}\right\rangle\left\langle A_{\text {trial }(i)}|H| v_{1}\right\rangle}{\left(\left\langleA_{\text {trial } \left.\left.(i)|H| A_{\text {trial }(i)}\right\rangle-\lambda_{1}\right) P\left(A_{\text {trial }(i)}\right)}\right.\right.} \\
& +\lim _{N \rightarrow \infty} \frac{1}{N} \sum_{j \neq 1} \sum_{i} \frac{\left\langle w \mid v_{j}\right\rangle\left\langle v_{j}|H| A_{\text {trial }(i)}\right\rangle\left\langle A_{\text {trial }(i)}|H| v_{1}\right\rangle}{\left(\lambda_{j}-\lambda_{1}\right)\left(\left\langle A_{\text {trial }(i)}|H| A_{\text {trial }(i)}\right\rangle-\lambda_{1}\right) P\left(A_{\text {trial }(i))}\right)}
\end{aligned}
$$

for any fixed vector $|w\rangle$.

\section{Two-body bound state}

Before presenting the results of our calculations, we first summarize what we expect in the weak-coupling non-relativistic limit. For the two-body bound state we consider all two-particle Fock states with zero momentum,

$$
|n,-n\rangle=a_{n}^{\dagger} a_{-n}^{\dagger}|0\rangle .
$$

These states satisfy the orthogonality and normalization conditions

$$
\left\langle n^{\prime},-n^{\prime} \mid n,-n\right\rangle=\delta_{n, n^{\prime}}+\delta_{n,-n^{\prime}} .
$$


At lowest order in $\lambda$ the $: \phi^{6}$ : interaction is not relevant to the two-body bound state problem. The effective interaction Hamiltonian is therefore

$$
\begin{aligned}
H_{I} & =-\lambda: \phi^{4}: \\
& =-\frac{3 \lambda}{4 L} \sum_{n_{1}+n_{2}+n_{3}+n_{4}=0} \frac{a_{-n_{1}}^{\dagger} a_{-n_{2}}^{\dagger} a_{n_{3}} a_{n_{4}}}{\left(\mu^{2}+\frac{n_{1}^{2} \pi^{2}}{L^{2}}\right)^{1 / 4}\left(\mu^{2}+\frac{n_{2}^{2} \pi^{2}}{L^{2}}\right)^{1 / 4}\left(\mu^{2}+\frac{n_{3}^{2} \pi^{2}}{L^{2}}\right)^{1 / 4}\left(\mu^{2}+\frac{n_{4}^{2} \pi^{2}}{L^{2}}\right)^{1 / 4}} .
\end{aligned}
$$

In the non-relativistic limit we can approximate $H_{I}$ as

$$
H_{I}=-\frac{3 \lambda}{4 \mu^{2} L} \sum_{n_{1}+n_{2}+n_{3}+n_{4}=0} a_{-n_{1}}^{\dagger} a_{-n_{2}}^{\dagger} a_{n_{3}} a_{n_{4}} .
$$

It is convenient to define the position-space basis vectors

$$
|x\rangle=\frac{1}{\sqrt{2 L}} \sum_{n=0, \pm 1, \ldots} e^{i \frac{n \pi x}{L}}|n,-n\rangle .
$$

Since we are considering a periodic box,

$$
|x\rangle=|x+2 L\rangle
$$

and the position-space vectors satisfy

$$
\left\langle x^{\prime} \mid x\right\rangle=\sum_{j=0, \pm 1, \ldots}\left[\delta\left(x^{\prime}-x+2 j L\right)+\delta\left(x^{\prime}+x+2 j L\right)\right] .
$$

For any function $\Psi(x)$, we can define

$$
|\Psi\rangle=\int_{-L}^{L} d x \Psi(x)|x\rangle .
$$

If $|\Psi\rangle$ is to have unit norm, then we require

$$
\int_{-L}^{L} d x|\Psi(x)|^{2}=\frac{1}{2} .
$$

In our position-space representation of the two-particle subspace, we note that

$$
\sum_{n=0, \pm 1, \ldots} a_{n}^{\dagger} a_{n}|x\rangle=2|x\rangle
$$

and

$$
\sum_{n=0, \pm 1, \ldots} \frac{n^{2} \pi^{2}}{L^{2}} a_{n}^{\dagger} a_{n}|\Psi\rangle=-2\left|\frac{\partial^{2} \Psi}{\partial x^{2}}\right\rangle .
$$

Upon restricting our Hilbert space to the zero-momentum two-particle subspace, we can simplify the interaction Hamiltonian by the substitution

$$
\sum_{n_{1}+n_{2}+n_{3}+n_{4}=0} a_{-n_{1}}^{\dagger} a_{-n_{2}}^{\dagger} a_{n_{3}} a_{n_{4}} \rightarrow \sum_{n_{1}, n_{2}} a_{n_{1}}^{\dagger} a_{-n_{1}}^{\dagger} a_{n_{2}} a_{-n_{2}} .
$$


We observe that

$$
\sum_{n_{1}, n_{2}} a_{n_{1}}^{\dagger} a_{-n_{1}}^{\dagger} a_{n_{2}} a_{-n_{2}}|x\rangle=4 L \delta(x)|x\rangle .
$$

The full non-relativistic Hamiltonian,

$$
H=\sum_{n=0, \pm 1, \ldots}\left(\mu+\frac{n^{2} \pi^{2}}{2 \mu L^{2}}\right) a_{n}^{\dagger} a_{n}-\frac{3 \lambda}{4 \mu^{2} L} \sum_{n_{1}, n_{2}} a_{n_{1}}^{\dagger} a_{-n_{1}}^{\dagger} a_{n_{2}} a_{-n_{2}},
$$

can now be represented as a Schrödinger operator:

$$
(H-2 \mu) \Psi(x)=\left[-\frac{1}{\mu} \frac{\partial^{2}}{\partial x^{2}}-\frac{3 \lambda}{\mu^{2}} \delta(x)\right] \Psi(x) .
$$

We now consider the eigenvalue equation

$$
(H-2 \mu) \Psi(x)=E \Psi(x) .
$$

Using the ansatz

$$
\begin{aligned}
\Psi_{2}(x) & \propto e^{-b|x|}+c e^{+b|x|}, \\
b & >0,
\end{aligned}
$$

we find

$$
\begin{aligned}
c & =e^{-\frac{3 \lambda L}{\mu} \frac{1+c}{1-c}}, \\
b & =\frac{3 \lambda}{2 \mu} \frac{1+c}{1-c}, \\
E & =-\frac{b^{2}}{\mu} .
\end{aligned}
$$

When

$$
e^{-\frac{3 \lambda L}{\mu}}<<1
$$

we have

$$
\begin{aligned}
c & =e^{-\frac{3 \lambda L}{\mu}}\left(1+O\left(e^{-\frac{3 \lambda L}{\mu}}\right)\right), \\
b & =\frac{3 \lambda}{2 \mu}\left(1+O\left(e^{-\frac{3 \lambda L}{\mu}}\right)\right), \\
E & =-\frac{9 \lambda^{2}}{4 \mu^{3}}\left(1+O\left(e^{-\frac{3 \lambda L}{\mu}}\right)\right) .
\end{aligned}
$$

This expression for the binding energy is consistent with the derivation in [6]. In the infinite $L$ limit we obtain

$$
\Psi_{2}(x)=\sqrt{\frac{3 \lambda}{4 \mu}} e^{-\frac{3 \lambda|x|}{2 \mu}} .
$$




\section{Three-body bound state}

The analysis for the three-body bound state is slightly more involved. In the non-relativistic weak-coupling limit we restrict our attention to three-particle states with zero-momentum,

$$
\left|n_{1}, n_{2},-n_{1}-n_{2}\right\rangle=a_{n_{1}}^{\dagger} a_{n_{2}}^{\dagger} a_{-n_{1}-n_{2}}^{\dagger}|0\rangle .
$$

We again construct position-space basis states

$$
\left|x_{1}, x_{2}\right\rangle=\frac{\sqrt{3}}{2 L} \sum_{n_{1}, n_{2}=0, \pm 1, \ldots} e^{i \frac{n_{1} \pi x_{1}}{L}} e^{i \frac{n_{2} \pi x_{2}}{L}} e^{i \frac{\left(-n_{1}-n_{2}\right) \pi\left(-x_{1}-x_{2}\right)}{L}}\left|n_{1}, n_{2},-n_{1}-n_{2}\right\rangle,
$$

which satisfy the orthogonality and normalization conditions

$$
\left\langle x_{1}^{\prime}, x_{2}^{\prime} \mid x_{1}, x_{2}\right\rangle=\sum_{j, k=0, \pm 1, \ldots}\left[\begin{array}{c}
\delta\left(x_{1}^{\prime}-x_{1}+\left(\frac{2 j}{3}+2 k\right) L\right) \delta\left(x_{2}^{\prime}-x_{2}+\frac{2 j}{3} L\right) \\
+\left\{x_{1} \leftrightarrow x_{2} \leftrightarrow-x_{1}-x_{2}\right\}
\end{array}\right] .
$$

The periodicity of our system implies

$$
\begin{aligned}
\left|x_{1}, x_{2}\right\rangle & =\left|x_{1}+\frac{2}{3} L, x_{2}+\frac{2}{3} L\right\rangle \\
& =\left|x_{1}+2 L, x_{2}\right\rangle=\left|x_{1}, x_{2}+2 L\right\rangle .
\end{aligned}
$$

For any function $\Psi\left(x_{1}, x_{2}\right)$, we define

$$
|\Psi\rangle=\int_{R} d x_{1} d x_{2} d \Psi\left(x_{1}, x_{2}\right)\left|x_{1}, x_{2}\right\rangle,
$$

where $R$ is the region

$$
R=\left\{\begin{array}{c}
-L<x_{1} \leq L \\
-\frac{1}{3} L<x_{2} \leq \frac{1}{3} L
\end{array}\right.
$$

If $|\Psi\rangle$ is to have unit norm then we must have

$$
\int_{R} d x_{1} d x_{2}\left|\Psi\left(x_{1}, x_{2}\right)\right|^{2}=\frac{1}{6}
$$

We note that

$$
\sum_{n=0, \pm 1, \ldots} \frac{n^{2} \pi^{2}}{L^{2}} a_{n}^{\dagger} a_{n}|\Psi\rangle=-\frac{2}{3}\left|\frac{\partial^{2} \Psi}{\partial x_{1}^{2}}+\frac{\partial^{2} \Psi}{\partial x_{2}^{2}}-\frac{\partial^{2} \Psi}{\partial x_{1} \partial x_{2}}\right\rangle .
$$

With a little work we also find

$$
\begin{aligned}
& \sum_{n_{1}+n_{2}+n_{3}+n_{4}=0} a_{-n_{1}}^{\dagger} a_{-n_{2}}^{\dagger} a_{n_{3}} a_{n_{4}}\left|x_{1}, x_{2}\right\rangle \\
& =4 L \sum_{j}\left[\delta\left(x_{1}+2 x_{2}+2 j L\right)+\delta\left(2 x_{1}+x_{2}+2 j L\right)+\delta\left(x_{1}-x_{2}+2 j L\right)\right]\left|x_{1}, x_{2}\right\rangle .
\end{aligned}
$$


For the moment we will neglect the $: \phi^{6}:$ term. Later we will show that this term produces only a higher order correction in $\lambda$. The effective non-relativistic Hamiltonian,

$$
H=\sum_{n=0, \pm 1, \ldots}\left(\mu+\frac{n^{2} \pi^{2}}{2 \mu L^{2}}\right) a_{n}^{\dagger} a_{n}-\frac{3 \lambda}{4 \mu^{2} L} \sum_{n_{1}+n_{2}+n_{3}+n_{4}=0} a_{-n_{1}}^{\dagger} a_{-n_{2}}^{\dagger} a_{n_{3}} a_{n_{4}},
$$

can now be represented as a Schrödinger operator:

$$
\begin{aligned}
(H-3 \mu) \Psi & =-\frac{1}{3 \mu}\left(\frac{\partial^{2} \Psi}{\partial x_{1}^{2}}+\frac{\partial^{2} \Psi}{\partial x_{2}^{2}}-\frac{\partial^{2} \Psi}{\partial x_{1} \partial x_{2}}\right) \\
& -\frac{3 \lambda}{\mu^{2}} \sum_{j}\left[\begin{array}{c}
\delta\left(x_{1}+2 x_{2}+2 j L\right)+\delta\left(2 x_{1}+x_{2}+2 j L\right) \\
+\delta\left(x_{1}-x_{2}+2 j L\right)
\end{array}\right] \Psi .
\end{aligned}
$$

In the infinite $L$ limit we can write the energy eigenvalue equation as

$$
-\frac{1}{3 \mu}\left(\frac{\partial^{2} \Psi}{\partial x_{1}^{2}}+\frac{\partial^{2} \Psi}{\partial x_{2}^{2}}-\frac{\partial^{2} \Psi}{\partial x_{1} \partial x_{2}}\right)-\frac{3 \lambda}{\mu^{2}}\left[\begin{array}{c}
\delta\left(x_{1}+2 x_{2}\right)+\delta\left(2 x_{1}+x_{2}\right) \\
+\delta\left(x_{1}-x_{2}\right)
\end{array}\right] \Psi=E \Psi .
$$

Using the ansatz

$$
\Psi_{3} \propto e^{-b\left(\left|x_{1}+2 x_{2}\right|+\left|2 x_{1}+x_{2}\right|+\left|x_{1}-x_{2}\right|\right)},
$$

we find

$$
\begin{gathered}
b=\frac{3 \lambda}{2 \mu}, \\
E=-\frac{9 \lambda^{2}}{\mu^{3}} .
\end{gathered}
$$

¿From the normalization condition,

$$
\Psi_{3}=\frac{\sqrt{3} \lambda}{\mu} e^{-\frac{3 \lambda}{2 \mu}\left(\left|x_{1}+2 x_{2}\right|+\left|2 x_{1}+x_{2}\right|+\left|x_{1}-x_{2}\right|\right)} .
$$

We now return to the $: \phi^{6}:$ term. This term produces an interaction proportional to

$$
\frac{\lambda}{\mu^{3} L^{2}} \delta\left(x_{1}\right) \delta\left(x_{2}\right)
$$

¿From (51) we see that

$$
\left\langle\Psi_{3}\left|\delta\left(x_{1}\right) \delta\left(x_{2}\right)\right| \Psi_{3}\right\rangle=O\left(\lambda^{2}\right),
$$

and therefore the $: \phi^{6}:$ interaction produces an effect of order $O\left(\lambda^{3}\right)$. We expect however that the repulsive delta function will suppress $\Psi_{3}$ near $x_{1}=x_{2}=0$. 


\section{Results}

In Table 1 we show for several values of $\lambda$ the mass of the one-particle state, $m_{1}$, and the two-body bound state, $m_{2}$, which is the relativistic state that is continously connected to the non-relativistic two-body bound state. For comparison we show the data for $m_{1}$ and $m_{2}$ from the lattice diagonalization study of 阿 (BD), non-relativistic Schrödinger results derived above for infinite $L(\mathrm{~S})$ and $L=2.5 \pi \mu^{-1}(\mathrm{~L})$, and Gaussian effective potential results from [8, 9 ] (GEP). The remaining column of data comes from an exact diagonalization of the Hamiltonian restricted to two-particle Fock states (F). In order to obtain the ratio $m_{2} / m_{1}$ in this approximation we take $m_{1}=\mu$.

\begin{tabular}{|l|l|l|l|l|l|l|l|l|}
\hline$\lambda / \mu^{2}$ & $m_{1} / \mu$ & $\mathrm{BD}$ & $m_{2} / m_{1}$ & $\mathrm{~S}$ & $\mathrm{~L}$ & $\mathrm{~F}$ & $\mathrm{BD}$ & GEP \\
\hline 0.1 & $0.995(1)$ & 0.994 & $1.970(2)$ & 1.978 & 1.967 & 1.975 & 1.916 & 1.982 \\
\hline 0.2 & $0.975(5)$ & 0.972 & $1.92(1)$ & 1.910 & 1.907 & 1.934 & 1.821 & 1.938 \\
\hline 0.3 & $0.94(1)$ & 0.928 & $1.85(1)$ & 1.798 & 1.797 & 1.877 & 1.718 & 1.878 \\
\hline 0.4 & $0.88(2)$ & 0.850 & $1.76(4)$ & 1.640 & 1.640 & 1.808 & 1.613 & 1.807 \\
\hline 0.5 & $0.77(4)$ & 0.720 & $1.66(8)$ & 1.438 & 1.438 & 1.730 & 1.520 & 1.728 \\
\hline 0.6 & $0.61(4)$ & & $1.71(10)$ & 1.190 & 1.190 & 1.646 & & 1.642 \\
\hline
\end{tabular}

The error bars in Table 1 and throughout our discussion include estimated errors from Monte Carlo errors, higher order contributions in the stochastic error correction series expansion, and residual dependence on the ultraviolet cutoff $\Lambda$. Of these errors we find the higher order contributions in the expansion to be the largest source of error. Any follow-up study of this system should push the calculation to higher order or make use of the stochastic Lanczos method introduced in [2].

The results from [7] (BD) were carried out on a much smaller system. While their results for $m_{1}$ appear in agreement with ours, they have noted that their calculation for $m_{2}$ is considerably affected by the small system size. At small coupling at least our own finite size effects appear to be small, as is suggested by the agreement of the Schrödinger results S and L. For this range of couplings the GEP predictions are equivalent to the two-particle Fock state approximation (F) in the limit of infinite $L$ and $\Lambda$. This is because at these couplings $\bar{\Omega}=m_{1}=\mu$, where $\bar{\Omega}$ is the optimal GEP variational mass parameter as defined in 8 8].

As expected, our results agree with the Schrödinger results at small coupling. They also appear reasonably close to the two-particle Fock approximation, F, for all values of coupling. In our next calculation we measure how much of the bound state does in fact reside in the two-particle sector. Let $\left|m_{2}\right\rangle$ be the normalized bound state and $P_{2}$ be the projection operator onto the two-particle subspace. We define $N_{2}$ to be the norm of the two-particle projection

$$
N_{2}=\| P_{2}\left|m_{2}\right\rangle \| \text {. }
$$

\footnotetext{
${ }^{2}$ This is at least consistent since in the two-particle Fock approximation with normalordered interactions, the constituents of the bound state have no self-energy contributions.
} 
In Table 2 we show $N_{2}$ for the different couplings.

\begin{tabular}{|l|l|l|l|l|l|l|}
\hline \multicolumn{7}{|c|}{ Table 2} \\
\hline$\lambda / \mu^{2}$ & 0.1 & 0.2 & 0.3 & 0.4 & 0.5 & 0.6 \\
\hline$N_{2}$ & $0.9977(1)$ & $0.989(1)$ & $0.971(2)$ & $0.936(8)$ & $0.87(2)$ & $0.79(2)$ \\
\hline
\end{tabular}

We can pursue the question of the bound state wavefunction further. Let

$$
\Psi_{2}(x)=\frac{1}{N_{2}}\left\langle x \mid m_{2}\right\rangle,
$$

where $|x\rangle$ is the two-particle position state defined in (14). In Figures 1-3 we compare the wavefunctions $\Psi_{2}$ with the corresponding approximate wavefunctions $\Psi_{2}^{\mathrm{S}}, \Psi_{2}^{\mathrm{L}}, \Psi_{2}^{\mathrm{F}}$ for coupling values $\lambda=0.1 \mu^{2}, 0.2 \mu^{2}, 0.4 \mu^{2}$.

In our analysis we did not consider bound states beyond $\lambda=0.6 \mu^{2}$. This is because our diagonalization/Monte Carlo results indicate a first order phase transition near $\lambda=0.73 \mu^{2}$, where $\phi$ develops a vacuum expectation value

$$
\langle\phi\rangle=1.02(2) .
$$

As a matter of fact, there appears to be no bound state in the broken symmetry phase, and the two-body and three-body bound states in the symmetric phase are stable all the way up to the transition point. The phase transition can be seen quite clearly in the behavior of the energy levels. In Table 3 we show the lowest two energy levels $E_{0}$ and $E_{2}$ in the even $\phi$ sector. We see what appears to be a metastable vacuum at energy $E_{2}$ for $\lambda<0.73 \mu^{2}$ becoming the true vacuum at energy $E_{0}$ for $\lambda>0.73 \mu^{2} \cdot{ }^{3}$

\begin{tabular}{|l|l|l|}
\hline \multicolumn{3}{|c}{ Table 3} \\
\hline$\lambda / \mu^{2}$ & $E_{0}$ & $E_{2}$ \\
\hline 0.70 & $-0.37(5)$ & $0.09(10)$ \\
\hline 0.71 & $-0.41(5)$ & $-0.15(15)$ \\
\hline 0.72 & $-0.40(5)$ & $-0.29(20)$ \\
\hline 0.73 & $-0.49(20)$ & $-0.42(5)$ \\
\hline 0.74 & $-0.70(20)$ & $-0.42(5)$ \\
\hline
\end{tabular}

In Table 4 we show for several values of $\lambda$ the mass of the three-body bound state, $m_{3}$, which is the relativistic state that is continously connected to the non-relativistic three-body bound state. We were not able to find any previous studies of this state in the literature. For comparison we show data from the Schrödinger equation at infinite $L(\mathrm{~S})$ and two different Fock space approximations, $\mathrm{F}_{1}$ and $\mathrm{F}_{2}$. $\mathrm{F}_{1}$ corresponds with an exact diagonalization of the Hamiltonian restricted to three-particle Fock states, and $\mathrm{F}_{2}$ corresponds to the same thing, except with the $: \phi^{6}$ : interaction turned off.f To obtain the

\footnotetext{
${ }^{3}$ Since we are at finite volume the levels $E_{0}$ and $E_{2}$ come close but never actually become degenerate.

${ }^{4}$ This is an artificial approximation. Without the restriction to three-particle Fock states, turning off the : $\phi^{6}$ : interaction would make the theory unbounded below.
} 
ratio $m_{3} / m_{1}$ in the two Fock approximations we again take $m_{1}=\mu$.

\begin{tabular}{|l|l|l|l|l|}
\hline$\lambda / \mu^{2}$ & $m_{3} / m_{1}$ & $\mathrm{~S}$ & $\mathrm{~F}_{1}$ & $\mathrm{~F}_{2}$ \\
\hline 0.1 & $2.931(2)$ & 2.910 & 2.940 & 2.919 \\
\hline 0.2 & $2.83(1)$ & 2.640 & 2.862 & 2.770 \\
\hline 0.3 & $2.72(2)$ & 2.190 & 2.769 & 2.568 \\
\hline 0.4 & $2.59(6)$ & 1.560 & 2.667 & 2.336 \\
\hline 0.5 & $2.54(8)$ & 0.75 & 2.558 & 2.084 \\
\hline 0.6 & $2.62(15)$ & $<0$ & 2.444 & 1.818 \\
\hline
\end{tabular}

We notice that the Schrödinger approximation $(\mathrm{S})$ is not quite as accurate for given $\lambda$ as it was for the two-body bound state. Nevertheless the Fock approximation $\mathrm{F}_{1}$ still appears to follow the actual data relatively well. We observe that $m_{3}$ is not only below the $3 m_{1}$ continuum threshold but also below the $m_{2}+m_{1}$ threshold. The corresponding state therefore has a sensible interpretation as a stable particle.

In analogy with before, we let $\left|m_{3}\right\rangle$ be the normalized three-body bound state and $P_{3}$ be the projection operator onto the three-particle subspace. We define $N_{3}$ to be the norm of the three-particle projection.

$$
N_{3}=\| P_{3}\left|m_{3}\right\rangle \| .
$$

In Table 5 we show $N_{3}$ for different couplings.

\begin{tabular}{|l|l|l|l|l|l|l|}
\hline$\lambda / \mu^{2}$ & 0.1 & 0.2 & 0.3 & 0.4 & 0.5 & 0.6 \\
\hline$N_{3}$ & $0.9966(2)$ & $0.981(2)$ & $0.950(5)$ & $0.89(1)$ & $0.80(2)$ & $0.66(4)$ \\
\hline
\end{tabular}

Let

$$
\Psi_{3}\left(x_{1}, x_{2}\right)=\frac{1}{N_{3}}\left\langle x_{1}, x_{2} \mid m_{3}\right\rangle .
$$

In Figures 4-11 we compare the wavefunction $\Psi_{3}$ with the corresponding approximate wavefunctions $\Psi_{3}^{\mathrm{S}}, \Psi_{3}^{\mathrm{F}_{1}}, \Psi_{3}^{\mathrm{F}_{2}}$ for $\lambda=0.1 \mu^{2}$ and $0.4 \mu^{2}$. For visual clarity we plot the wavefunctions over three copies of the fundamental region $R$. We use the variables

$$
\begin{aligned}
& y_{1}=\frac{3}{2 L} x_{1}, \\
& y_{2}=\frac{\sqrt{3}}{2 L}\left(x_{1}+2 x_{2}\right),
\end{aligned}
$$

to make clear the hexagon symmetry. To guide the eye we have drawn dotted lines along the points

$$
\begin{aligned}
& x_{1}=x_{2}, \\
& x_{1}=-x_{1}-x_{2}, \\
& x_{2}=-x_{1}-x_{2} .
\end{aligned}
$$


These corresponds with configurations where two of the three constituents lie at the same point.

We notice that for both $\Psi_{3}$ and $\Psi_{3}^{\mathrm{F}_{1}}$, the inclusion of the repulsive : $\phi^{6}$ : interaction produces a marked suppression of the wavefunction near the origin. By independently changing the coefficients of : $\phi^{6}:$ and $: \phi^{4}:$, we have a simple laboratory to study the competition between two-body and three-body forces in three-body bound states, a topic of some relevance and recent interest in the triton system. 10

\section{Summary}

We have considered the relativistic bound states of $\phi^{6}-\phi^{4}$ theory in $1+1$ dimensions. Using a recently proposed diagonalization/Monte Carlo computational scheme, we calculated the two-body and three-body bound state energies and wavefunctions. The initial diagonalization was performed using the quasi-sparse eigenvector method, and the subsequent stochastic error correction was calcu-

lated to first-order using the series method. We made detailed comparisons of our numerical results with the available literature and several approximations, including non-relativistic weak-coupling solutions and various truncated Fock space approximations.

Applications to more complicated higher dimensional theories can be done in the same manner but require larger QSE subspaces and going beyond the first-order term in the series method or use of the stochastic Lanczos method. But even from the simple examples presented here, the potential for studying relativistic bound states with diagonalization/Monte Carlo methods is apparent.

Acknowledgments Financial support provided by the DFG and NSF.

\section{References}

[1] D. J. Lee, N. Salwen, D. D. Lee, hep-th/0002251.

[2] D. Lee, N. Salwen, M. Windoloski, hep-lat/0010039.

[3] J. Glimm, A. Jaffe, T. Spencer, Constructive quantum field theory, Proc. 1973 Ettore Majorana Inter. School of Math. Phys. (Springer, Berlin, 1973) 165.

[4] D. Lee, cond-mat/0008457; N. Salwen, cond-mat/0008458.

[5] P. Marrero, E. Roura, D. Lee, Phys. Lett. 471B (1999) 45.

[6] J. Dimock, J.-P. Eckmann, Commun. Math. Phys. 51 (1976) 41.

[7] T. Barnes, G. Daniell, Phys. Lett. 142B (1984) 188; Phys. Rev. D28 (1983) 2045. 


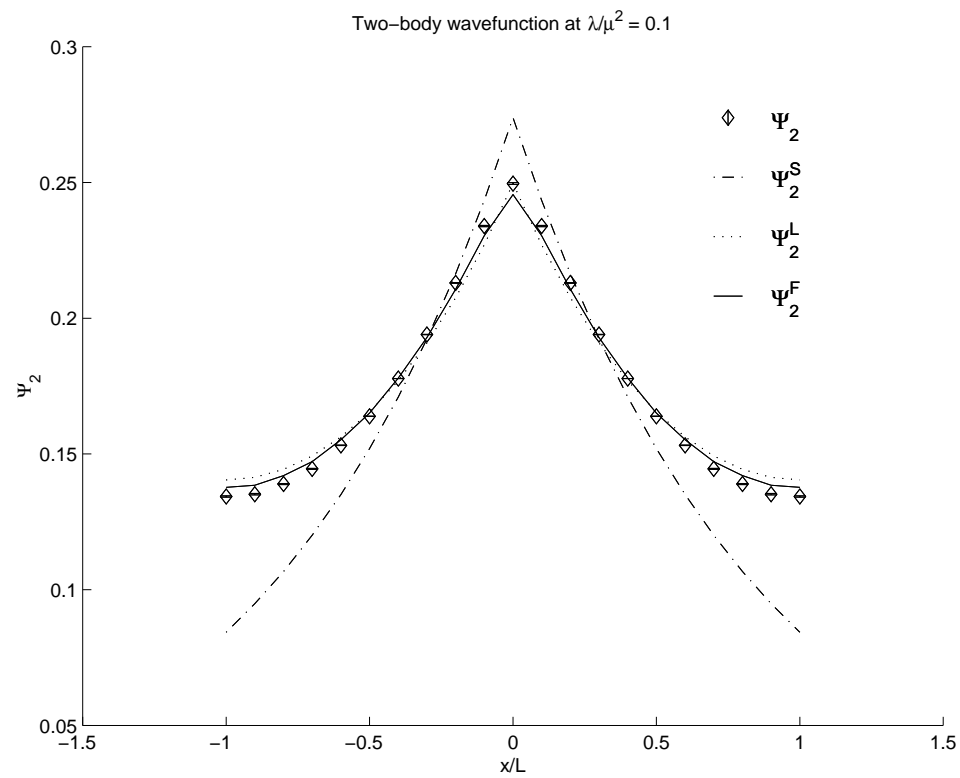

Figure 1: Two-body wavefunction $\Psi_{2}$ and approximate wavefunctions $\Psi_{2}^{\mathrm{S}}, \Psi_{2}^{\mathrm{L}}, \Psi_{2}^{\mathrm{F}}$ at $\lambda=0.1 \mu^{2}$.

[8] J. Darewych, M. Horbatsch, R. Koniuk, Phys. Rev. Lett. 54 (1985) 2188.

[9] P. Stevenson, Phys. Rev. D30 (1984) 1712; P. Stevenson, Phys. Rev. D32 (1985) 1389; P. Stevenson, I. Roditi, Phys.Rev. D33 (1986) 2305.

[10] P. Bedaque, H.-W. Hammer, U. van Kolck, Nucl. Phys. A676 (2000) 357. 


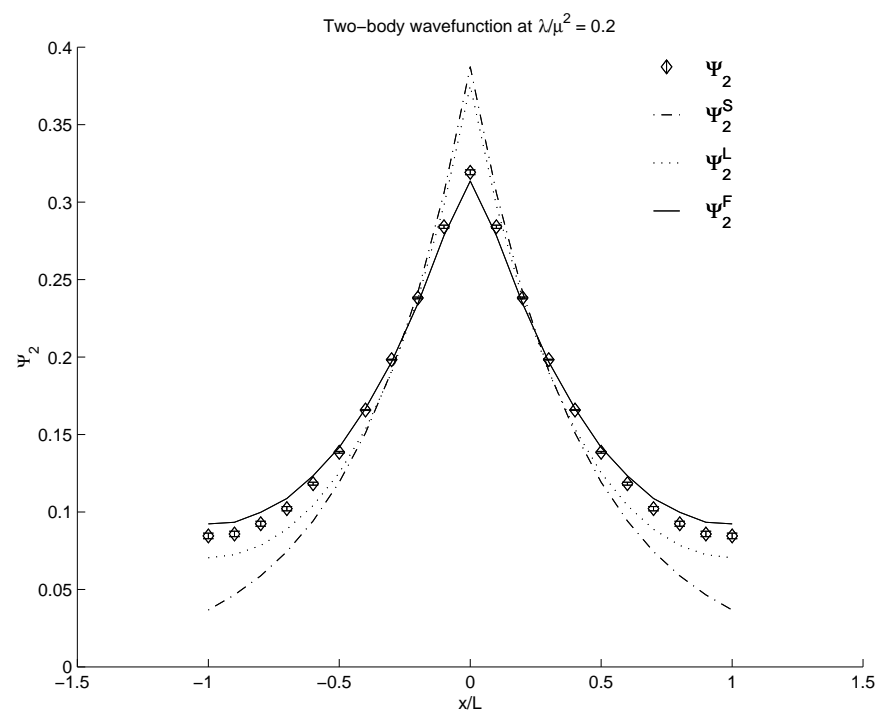

Figure 2: Two-body wavefunction $\Psi_{2}$ and approximate wavefunctions $\Psi_{2}^{\mathrm{S}}, \Psi_{2}^{\mathrm{L}}, \Psi_{2}^{\mathrm{F}}$ at $\lambda=0.2 \mu^{2}$.

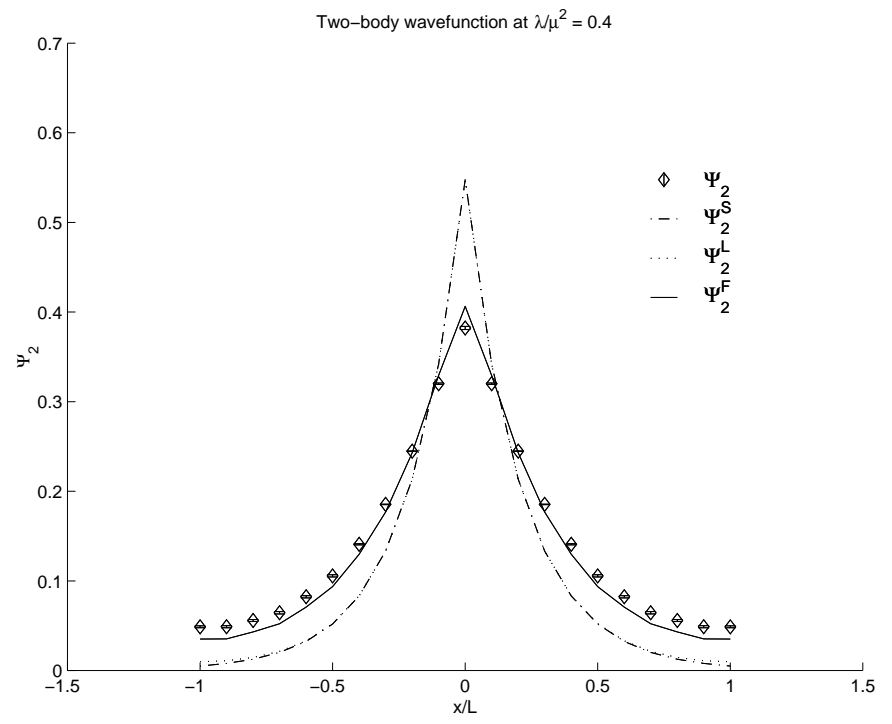

Figure 3: Two-body wavefunction $\Psi_{2}$ and approximate wavefunctions $\Psi_{2}^{\mathrm{S}}, \Psi_{2}^{\mathrm{L}}, \Psi_{2}^{\mathrm{F}}$ at $\lambda=0.4 \mu^{2}$. 
Three-body wavefunction $\Psi_{3}$ at $\lambda / \mu^{2}=0.1$

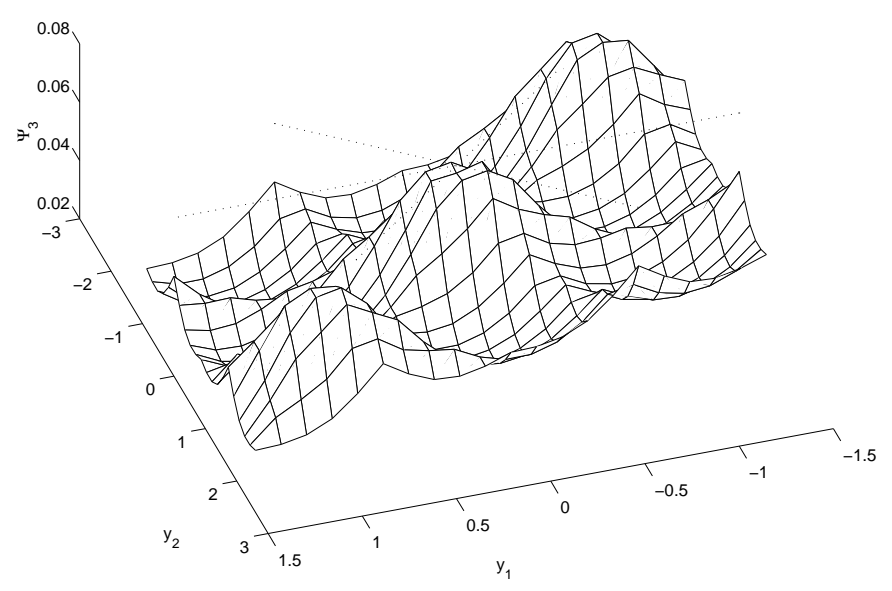

Figure 4: Three-body wavefunction $\Psi_{3}$ at $\lambda=0.1 \mu^{2}$.

Three-body wavefunction $\Psi_{3}^{S}$ at $\lambda / \mu^{2}=0.1$

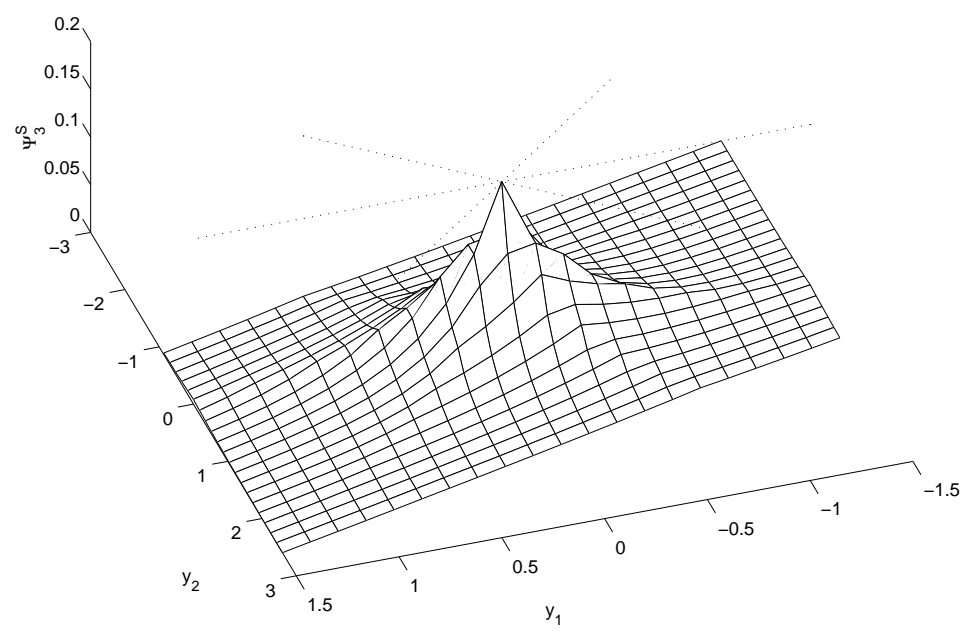

Figure 5: Approximate three-body wavefunction $\Psi_{3}^{\mathrm{S}}$ at $\lambda=0.1 \mu^{2}$. 
Three-body wavefunction $\Psi_{3^{1}}^{F}$ at $\lambda / \mu^{2}=0.1$

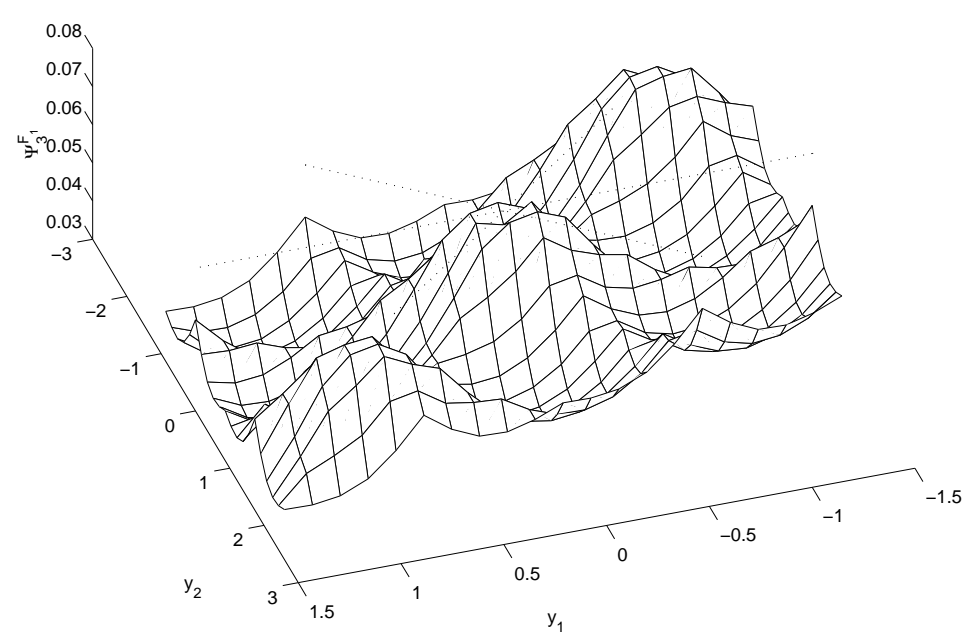

Figure 6: Approximate three-body wavefunction $\Psi_{3}^{\mathrm{F}_{1}}$ at $\lambda=0.1 \mu^{2}$.

Three-body wavefunction $\Psi_{3^{2}}^{F_{2}}$ at $\lambda / \mu^{2}=0.1$

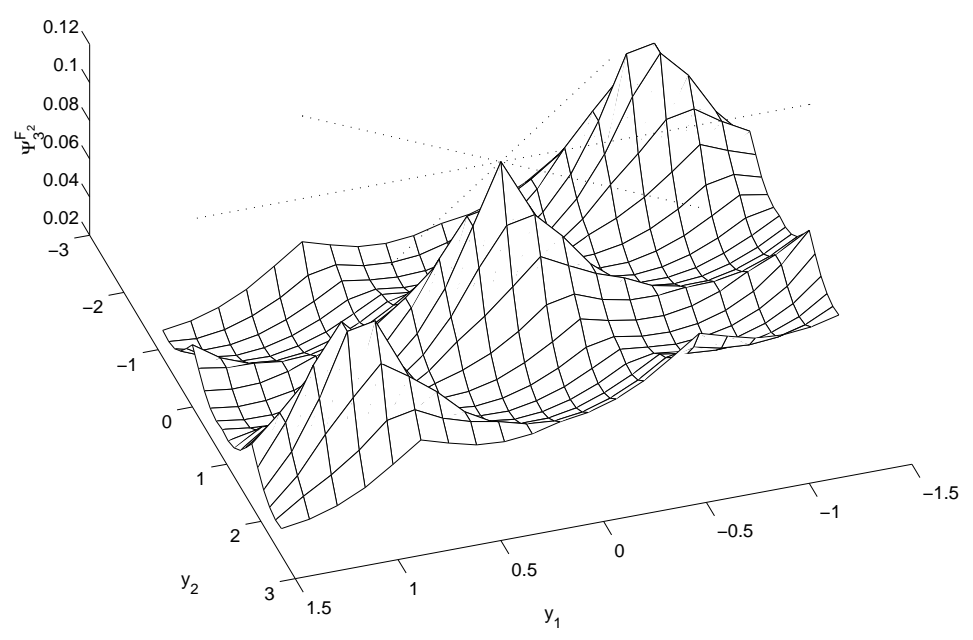

Figure 7: Approximate three-body wavefunction $\Psi_{3}^{\mathrm{F}_{2}}$ at $\lambda=0.1 \mu^{2}$. 
Three-body wavefunction $\Psi_{3}$ at $\lambda / \mu^{2}=0.4$

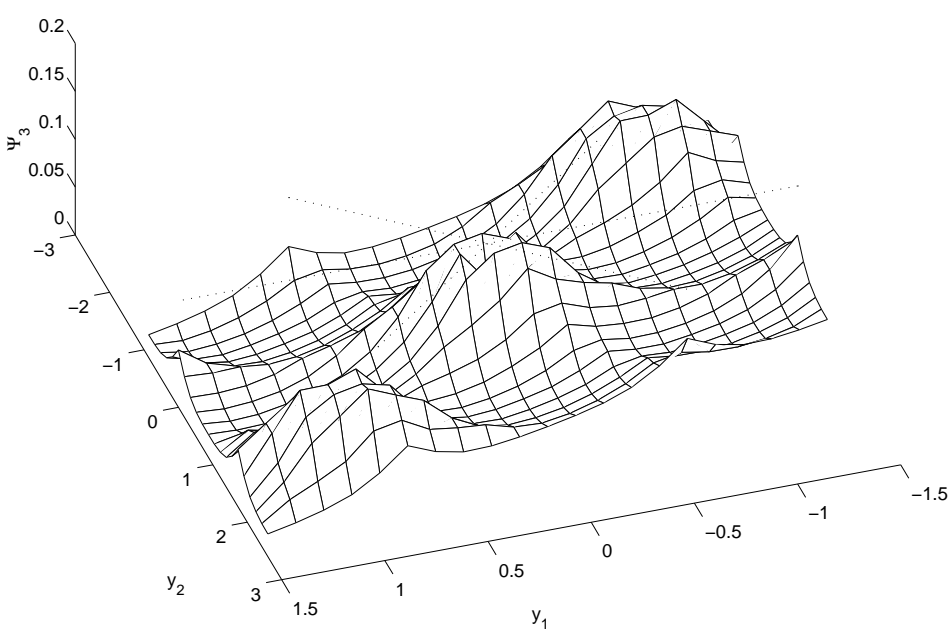

Figure 8: Three-body wavefunction $\Psi_{3}$ at $\lambda=0.4 \mu^{2}$.

Three-body wavefunction $\Psi_{3}^{S}$ at $\lambda / \mu^{2}=0.4$

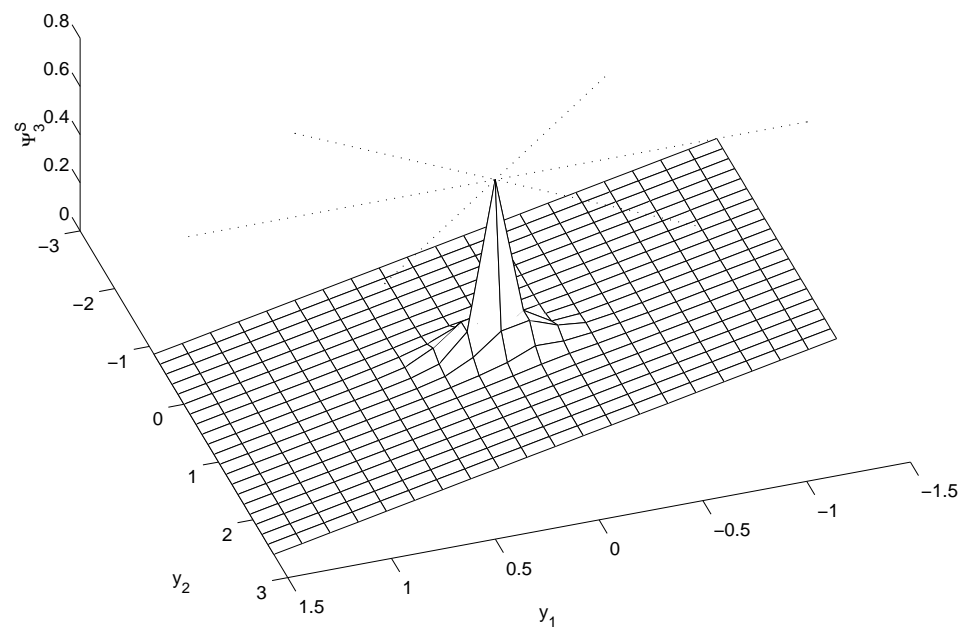

Figure 9: Approximate three-body wavefunction $\Psi_{3}^{\mathrm{S}}$ at $\lambda=0.4 \mu^{2}$. 
Three-body wavefunction $\Psi_{3^{1}}^{F_{1}}$ at $\lambda / \mu^{2}=0.4$

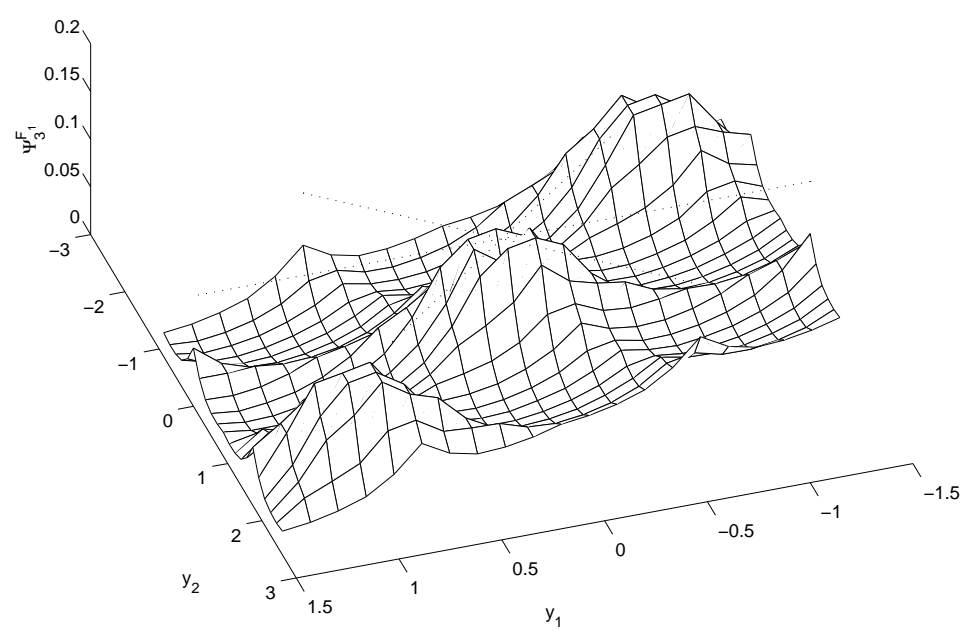

Figure 10: Approximate three-body wavefunction $\Psi_{3}^{\mathrm{F}_{1}}$ at $\lambda=0.4 \mu^{2}$.

Three-body wavefunction $\Psi_{3^{2}}^{F_{2}}$ at $\lambda / \mu^{2}=0.4$

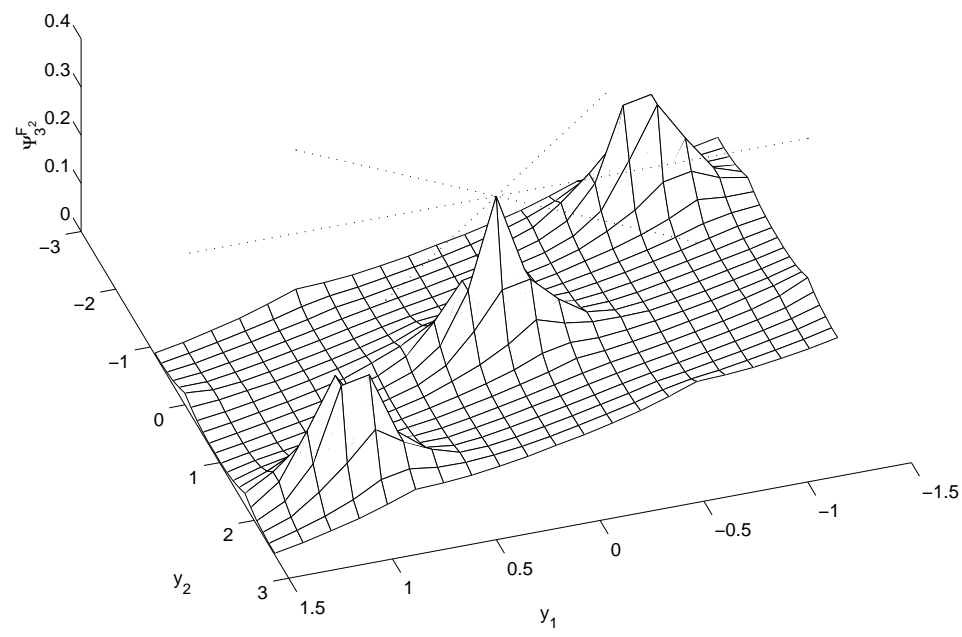

Figure 11: Approximate three-body wavefunction $\Psi_{3}^{\mathrm{F}_{2}}$ at $\lambda=0.4 \mu^{2}$. 